\title{
High-Speed and Low-Noise Multichannel system for Broadband Coherent Raman Imaging
}

\author{
A. Ragni, G. Sciortino, M. Sampietro, G. Ferrari \\ Dipartimento di Elettronica, Informazione e Bioingegneria \\ Politecnico di Milano, Milano, Italy \\ Email: andrea.ragni@polimi.it
}

\begin{abstract}
Broadband coherent Raman is a non-invasive and non-destructive spectroscopic technique with growing applications in the analysis of molecules and biological compounds.

This paper presents the first 32-ch modular platform for highspeed Broadband Raman Imaging, able to simultaneously acquire and process 32 wavelengths of the spectrum with a multichannel pseudo-differential lock-in structure for the compensation of the excess noise given by the laser source. The system is based on a custom integrated CMOS front-end, specifically designed for Broadband Raman applications, and a Xilinx Artix-7 FPGA for the parallel acquisition and real-time data elaboration. Experimental results show that the system is able to reach the shot noise limit and acquire a Raman image with a pixel dwell time of $100 \mu \mathrm{s}$.
\end{abstract}

\section{INTRODUCTION}

Every biological sample, from cell down to the single organic molecule, is characterized by a specific vibrational spectrum (also called Raman spectrum or fingerprint) which is a function of its anatomy and molecular structure. Raman spectroscopy is commonly used in materials science and biology to determine the chemical species of the sample by analyzing the Raman spectrum in the range $100 \mathrm{~cm}^{-1}-3500 \mathrm{~cm}^{-1}$. Several scientific papers demonstrate how this spectral region is highly informative for cancer differentiation, for instance the region $800-1800 \mathrm{~cm}^{-1}$ for gastric tumor [1] and $800-3100 \mathrm{~cm}^{-1}$ for brain tumor [2]. This technique is label-free and non-contact since the sample only interacts with two coherent pulsed lasers of different wavelengths in the near-infrared region (NIR), called Pump and Stokes. The Pump excites the molecules to a virtual state, the Stokes enhances the relaxation transition to a lower energy state. By using a broadband laser, consisting of sub-20fs pulses, is possible to simultaneously excite several resonant modes of molecules. If each fraction of the broadband spectrum is correctly detected with a photodiode array and elaborated in parallel with a multichannel electronics, a faster acquisition is possible (theoretically up to video-rate) because the whole Raman signature is acquired with only one optical measurement. However, the splitting of the optical on many photodiodes reduces the available signal posing severe requirements on the front-end electronics. Moreover, the femtosecond laser shows an excess noise order of magnitude of the theoretical shot noise [3]. Here, we present the first 32-channel acquisition and processing system enabling a fast broadband Raman spectroscopy and imaging of biological samples. Thanks to a fast Raman Imaging, more accurate data for the diagnosis will help determine the appropriate treatment

\author{
A. De La Cadena, F. Vernuccio, G. Cerullo, D. Polli \\ Dipartimento di Fisica \\ Politecnico di Milano, Milano, Italy \\ Email: dario.polli@polimi.it
}

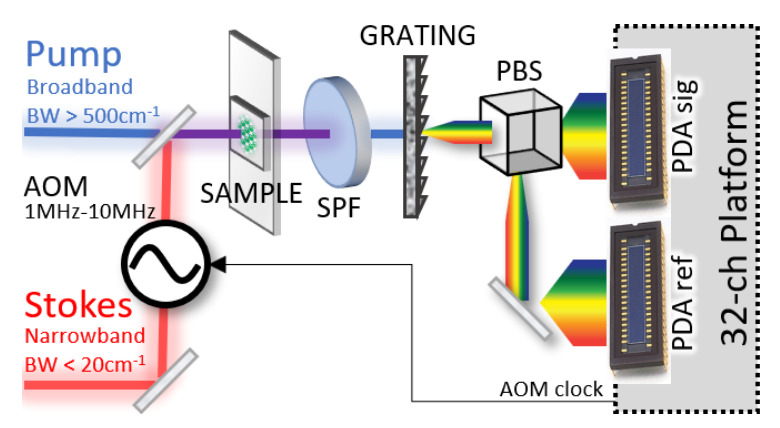

Fig. 1. Simplified block diagram of the In-line balanced detection [4] adopted in the project (narrowband Stokes and Broadband Pump).

for the tumour, furthermore, neoplasms can be located and their borders with normal tissue traced for surgery.

\section{RAMAN MICROSCOPE ARCHITECTURE OVERVIEW}

We adopted the lock-in technique as a detection scheme because it can improve performance for two main reasons: i) the spectrum of the Laser noise tends to decrease monotonically with frequency; ii) the laser average power is 3 to 5 orders of magnitude greater than the superimposed Raman signal which has to be measured (i.e. Offsets, drift and flicker noise would affect the Raman). This justifies the adoption of the lock-in technique for the detection of the Raman signal. The requirement of a fast acquisition of each pixel $\left(t_{d w e l l}\right.$ $\leq 100 \mu \mathrm{s}$ ) has imposed a parallel readout of 32 wavelengths, that is the development of 32 lock-in amplifiers. In order to obtain a compact and efficient platform, the front-end amplifiers and the lock-in demodulation have been embedded in specifically designed integrated circuits. The main characteristics required from the front-end for this application are: i) scalable up to 32 channels; ii) lock-in based to detect the weak Raman signal (10ppm to 1000ppm) in the frequency range of $1 \mathrm{MHz}-10 \mathrm{MHz}$; iii) an electronic noise below to the unavoidable shot noise of the photodetectors; iv) differential structure for laser intensity noise compensation; v) Separated outputs for DC (proportional to the laser average power) and $\mathrm{AC}$ (proportional to the Raman signal) needed for Raman normalization; vi) Compact structure. Figure 1 shows the simplified block diagram of the Optical setup based on the Inline balanced detection (IBD) [4] with Broadband Pump. The 
commercial Yb-fiber laser "Fidelity coherent HP" produces the main beam (also called "Fundamental") consisting of laser pulses with wavelength equal to $1040 \mathrm{~nm}, 140 \mathrm{fs}$ pulse duration and repetition frequency of $80 \mathrm{MHz}$. This main beam is split into two branches: a narrowband Stokes and a broadband Pump. A time-delayed replica of the broadband Pump is generated to be used as a reference. This allows to compensate the laser common mode fluctuations with a balanced detection. Thanks to the IBD technique, the two replicas cross the sample in the same position and in this way they can maintain their balance during image acquisition making the common mode noise compensation more effective. The narrowband Stokes is optically modulated between $1 \mathrm{MHz}$ and $10 \mathrm{MHz}$ with an acousto optic modulator (AOM) placed before the sample. The Stokes has the same polarization and delay of one Pump replica (i.e. temporally overlapped), thus only here a modulated Raman scattering can occur. Successively, the two broadband replicas (one containing the modulated Raman and the Reference) are first dispersed with a diffraction grating, then get spatially separated by a polarizing beam splitter (PBS) and finally focused onto two photodiode arrays of the custom multichannel lock-in system. Each photodiode of the array acquires a single portion (i.e. a small wavelength range) of the broadband spectrum therefore the total laser power is divided between all the elements of the arrays. The narrowband Stokes, having no information, is blocked after the sample with a SP (Short Pass) filter.

\section{HARDWARE IMPLEMENTATION}

Figure 2 shows the architecture of the system presented in this work. The adopted differential stage and mixer are analog circuits to overcome the resolution limit of digital LIA [5]. Moreover, the implementation of 32 digital lock-in, operating in the $\mathrm{MHz}$ range, would require too many resources. This system with 32 lock-in channels is composed by:

- 8 MODULES: each operating one ASIC. This, specifically designed for Broadband Raman applications, includes four pseudo-differential lock-in acquisition channels. The single module also contains: the ADC (AD7616) for the 4-ch acquisition, DAC (AD5668) for the equalization stage control voltages, output stages after the custom IC and its own independent power supply protected with a voltage supervisor;

- MOTHERBOARD: where the 8 modules are installed with PCI Express connectors. On the motherboard is also present: an additional ADC and DAC to control the $\mathrm{X}-\mathrm{Y}-\mathrm{Z}$ movement of the microscope, the Xilinx Artix7 FPGA on the Opal Kelly XEM7310 module (for the 32 channels control and USB communication) and the two photodiode arrays (PDAs) for signal and reference. These are mounted in the motherboard because the $2 \times 32$ photodiodes are connected to the pseudo-differential lockin amplifiers distributed on 8 modules $(2 \times 4$ on each);

- MAIN POWER SUPPLY BOARD: compact and efficient power supply based on DC-DC converters. It is installed on the motherboard and it can deliver the $20 \mathrm{~W}$ required to power the whole system, including the 8 modules and the FPGA. Thanks to this board, the system can be powered with just a standard laptop power supply (or laptop charger) of $16-20 \mathrm{~V}$ and power greater than $40 \mathrm{~W}$.

The choice for the $P D A_{\text {sig }}$ and $P D A_{\text {ref }}$ (Photodiode Array) sensors fell on the model A5C-35 produced by OSI Optoelectronics. Built in a DIP40 package, this sensor contains 35 silicon photodiodes laid adjacent to each other forming a one-dimensional sensing area on a common cathode substrate. Each element has a size of $4.39 \times 0.89 \mathrm{~mm}$, about $3.9 \mathrm{~mm}^{2}$ of active area, and a spacing between two adjacent of only $0.1 \mathrm{~mm}$. This dimensions are consistent with the optical system requirements to relax the alignment. The common PDA cathode is connected to $+15 \mathrm{~V}$ to minimize the junction capacitance $\left(C_{p d}=12 \mathrm{pF}\right)$ while each photodiode anode is connected to the input node of the custom IC. This has been designed in $0.35 \mu \mathrm{m}$ AMS CMOS technology and occupies an area of $3.56 \times 1.82 \mathrm{~mm}^{2}$ (including a test channel) and has a $3.3 \mathrm{~V}$ supply voltage with $21 \mathrm{~mA}$ current consumption per channel. The full details of the chip will be present elsewhere. Briefly, the first amplification stage consists of an advanced TIA made with an integrator stage (GBWP $\approx 1 \mathrm{GHz}$, decompensated) and a feedback network $\mathrm{H}(\mathrm{s})$ used to manage the DC. The first-stage is designed with a high bandwidth and a programmable gain to handle an average optical power on the photodiode $\left(P_{p d}\right)$ from $50 \mu \mathrm{W}$ to $600 \mu \mathrm{W}$ without saturating while minimizing the noise. Having the need to normalize the received signal to get the Raman gain, the amplifying stage can separately manage the average value of the pulse-train laser and the amplitude of the modulated signal (Raman): in other words, the DC $(<500 \mathrm{kHz})$ and $\mathrm{AC}(>500 \mathrm{kHz})$ components. The second stage includes an innovative auto-balancing network to automatically compensate the amplitude of the signal and reference voltages, independently. This feature enables to increase the CMRR of the differential structure and better compensate the common mode laser intensity fluctuations with the following fully differential subtractor. The auto-balancing network equalizes the peak amplitude of the signals by properly controlling a variable gain amplifier on the reference path. Since the Raman signal is commonly less than few thousands of ppm, it introduces a negligible error on the equalization allowing an effective reduction of the commonmode noise given by the laser. The outputs of the subtractor are connected to a CMOS double balanced mixer to perform the lock-in acquisition (i.e. the demodulation) of the Raman signal. The mixer is composed by four switches (transmission gates), driven by non-overlapped square wave clocks at the modulation frequency $1-10 \mathrm{MHz}$. The mixer outputs, with the Raman demodulated down to baseband, are connected to the output pads through a pair of resistors $R_{s}=20 \mathrm{k} \Omega$. These, with the external capacitor $C_{s}=68 \mathrm{pF}$, realize a first passive low-pass filter with cutoff frequency $f_{c} \approx 58 \mathrm{kHz}$. The balanced output of the four CMOS mixers, inside the custom IC, are connected to four INAs AD8226 (by Analog Devices) with $G=10$ to effectively amplify the differential signal (i.e. Raman signal). 


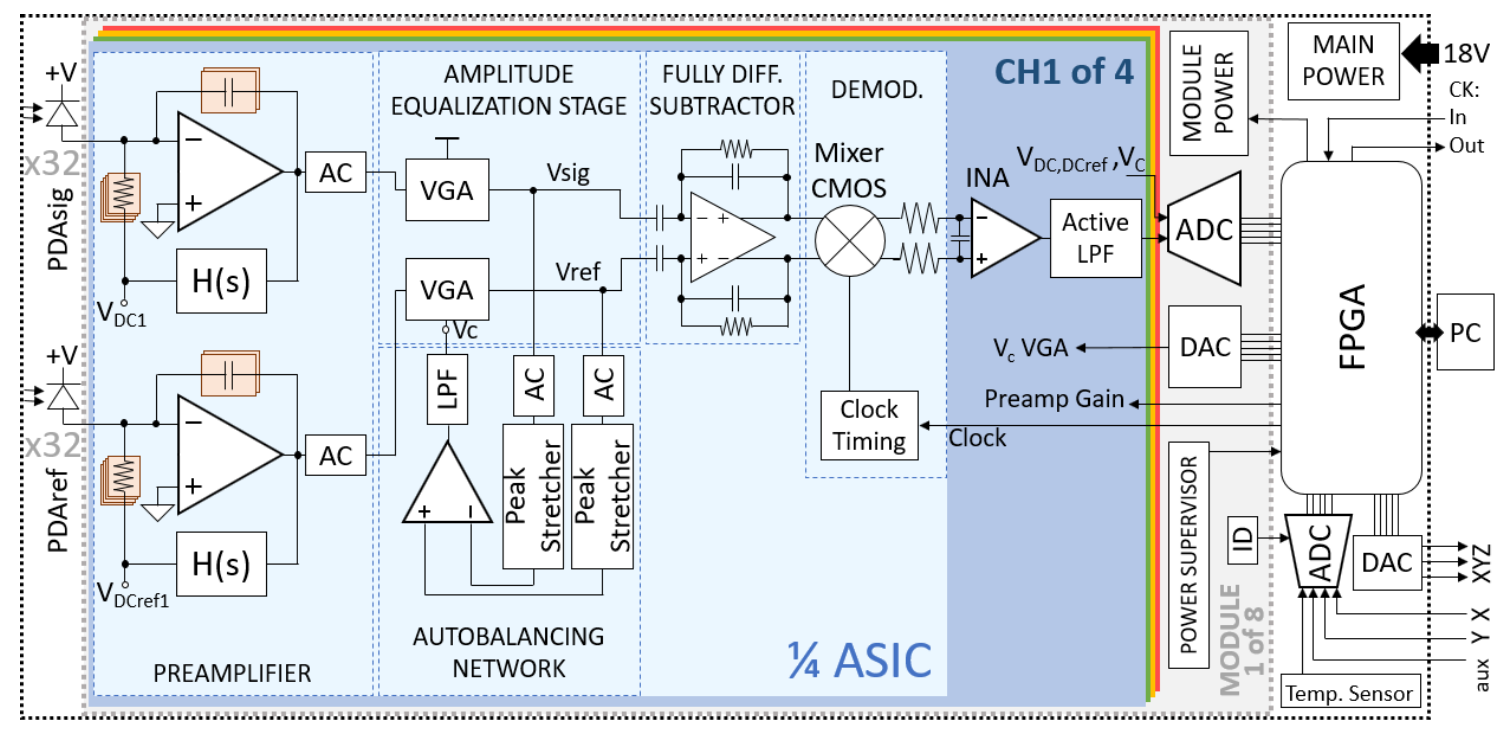

Fig. 2. Block diagram of the 32-ch platform with highlighted one channel (out of 4) of the custom chip (ASIC), the logic on the module (1 out of 8 ) and the FPGA on the motherboard.

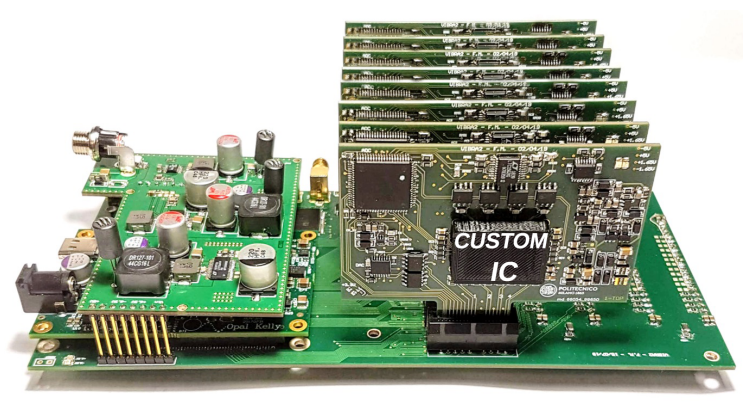

Fig. 3. Photo of the 32-ch system composed by 8 modules installed on the motherboard with PCIe connectors, main power supply board and the Opal Kelly module below. The two PDAs are on the bottom of the motherboard.

The four INA outputs are connected to a compact Quad active low-pass filter made with the IC LTC1562 (by Linear Technology). This component, requiring only few external resistors and capacitors, allows to implement four independent $2^{\text {nd }}$ order LPF with desired cutoff frequency, $16 \mathrm{kHz}$ in this case. On each module, all the analog signals required for the Raman spectroscopy ( 8 proportional to the laser average power for signal and reference, 4 proportional to the demodulated Raman and other control signals) are converted with the 16-bit, 16-ch, DAS (Data Acquisition System) AD7616 (by Analog Devices). The FPGA is operated to acquire and elaborate the total of about $32 \mathrm{MB} / \mathrm{s}$ of data, coming from the 8 modules (one ADC per module), and transfer formatted packets to a PC using the USB 3.0 connection. The FPGA also generates a clock to drive the optical modulator and 8 clocks for the synchronous demodulation on each module. The delay between the clocks is tunable with a resolution of $2.5 \mathrm{~ns}$ in order to maximize the lock-in output. A modern user interface developed in C\# (C sharp) using Visual Studio is used to control the system through the FPGA (e.g. set the first stage gain, program the DAC/ADC etc.) and elaborate the received data stream to visualize the Raman image in real time.

\section{EXPERIMENTAL RESULtS}

The custom IC and the 32-ch system are fully characterized and validated with electrical signals first, then in a real optical setup for broadband Raman imaging. In particular, in the modulation range $1-10 \mathrm{MHz}$, the measured inputreferred current noise ranges approximately from $1.8 p \mathrm{~A} / \sqrt{\mathrm{Hz}}$ to $9 p A / \sqrt{H z}$ depending on the gain selection. In the input power range from $50 \mu \mathrm{W}$ to $600 \mu \mathrm{W}$, considering the photodiode responsivity $\mathrm{R}=0.5 \mathrm{~A} / \mathrm{W}$, the estimated input-referred shot-noise is $2.8-9.8 p \mathrm{~A} / \sqrt{\mathrm{Hz}}$. It results that a shot-noise limited measurement is possible in this power range. This is also verified in a real Raman experiment with the optical setup of Fig.1. The Raman spectra of 4 different solvents: Acetone, Ethanol, Methanol and Isopropanol are correctly acquired as shown in Fig.4. The measurement is shot-noise limit even with a laser average power of only $60 \mu \mathrm{W} /$ channel. For this power, the estimated shot-noise is $1.15 n W_{r m s}$ and the measured noise is $1.59 n W_{r m s}$ (both Input-referred). With an integration time of $44 \mu$ s the obtained sensitivity is lower than 10ppm. The acquired Raman spectra are in agreement with the results available in literature [6]. Finally, thanks to a piezo for the X-Y movement of the sample, a $512 \times 512$ pixels image is also acquired, practically at shot-noise limit (Input-referred estimated shot-noise: $1.15 n W_{r m s}$, Measured noise: $1.59 n W_{r m s}$ ), with an integration time of $10 \mu \mathrm{s}$ (Fig.5). The sample consists of microscopic beads of PS (Polystyrene, $10 \mu \mathrm{m}$ ) and PMMA (Polymethyl methacrylate, $5 \mu \mathrm{m}$ ) which are characterized by Raman peaks at different wavelength: $3066 \mathrm{~cm}^{-1}$ and $2956 \mathrm{~cm}^{-1}$, respectively. Each pixel of the image contains a 32 points Raman spectrum that allow the material identification. Total acquisition time is 26 seconds 

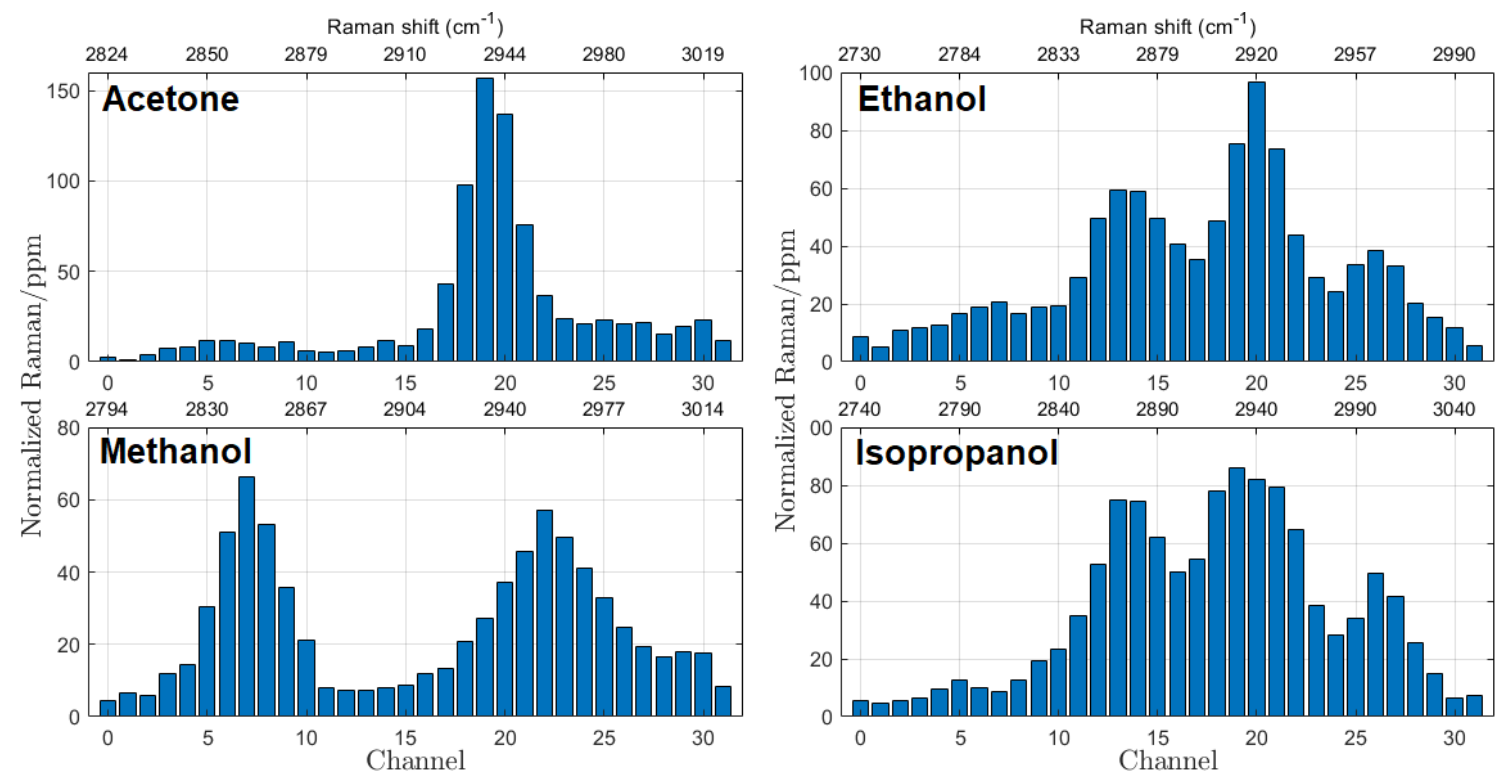

Fig. 4. Measured Raman spectra of four different solvents: Acetone, Ethanol, Methanol and Isopropanol. $P_{p d} \approx 60 \mu W$, Input-referred shot-noise: $1.18 n W_{r m s}$, Measured noise: $1.4 n W_{r m s}$ (considering balanced acquisition and assuming a photodiode responsivity $\mathrm{R}=0.5 \mathrm{~A} / \mathrm{W}$ ).

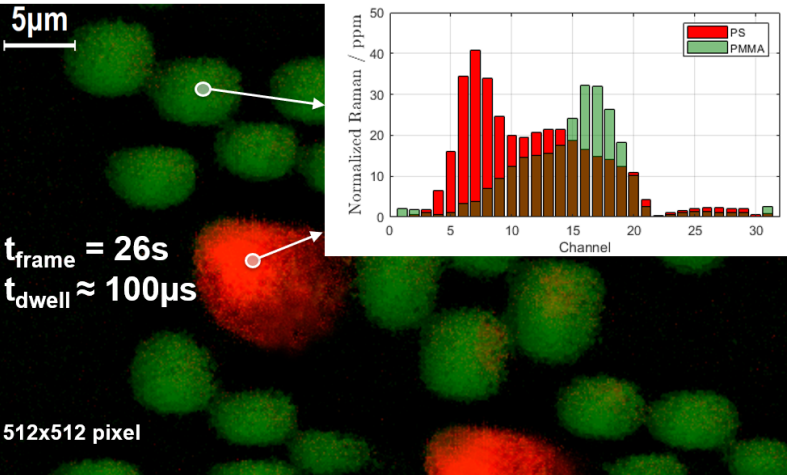

Fig. 5. Raman image (512x512 pixels) acquired with the 32-ch system. The plastic beads: PS (red, $10 \mu \mathrm{m}$ diameter) and PMMA (green, $5 \mu \mathrm{m}$ diameter) can be differentiated because of their different Raman spectrum. Parameters: $\tau=10 \mu s, P_{p d} \approx 50 \mu W, f_{m o d}=1.6 \mathrm{MHz}$ and $t_{d w e l l} \approx 100 \mu \mathrm{s}$. Inputreferred shot-noise: $1.15 n W_{r m s}$, Measured noise: $1.59 n W_{r m s}$.

resulting in a pixel dwell time $t_{d w e l l} \approx 100 \mu \mathrm{s}$. This demonstrates that the present work has the best performance in terms of resolution (for a given $t_{d w e l l}$ and a low laser power of $50 \mu \mathrm{W} / \mathrm{channel})$ when compared to the state-of-art ([7], [8], [9] and [10]).

\section{CONCLUSION}

In this paper, a novel high-speed and low-noise 32-ch modular system, for Broadband Raman Imaging, is presented and tested. Thanks to 8 custom integrated CMOS frontends and a Xilinx Artix-7 FPGA, 32 portions of the Raman spectrum can be acquired at shot noise limit with a time constant down to $10 \mu \mathrm{s}$ making this system the fastest of its kind. This is demonstrated by acquiring the Raman spectra of four different solvents and a 512x512 pixels Raman image (32 wavelengths/pixel) with a pixel dwell time of $100 \mu \mathrm{s}$. The laser common-mode noise is attenuated by the pseudo-differential lock-in structure and the measurement is practically shot-noise limited even with an average power of only $50 \mu \mathrm{W} / \mathrm{channel}$.

\section{REFERENCES}

[1] SK Teh, W Zheng, KY Ho, M Teh, KG Yeoh, and Z Huang. Diagnostic potential of near-infrared raman spectroscopy in the stomach: differentiating dysplasia from normal tissue. British journal of cancer, 98(2):457, 2008.

[2] Yan Zhou, Cheng-Hui Liu, Yi Sun, Yang Pu, Susie Boydston-White, Yulong Liu, and Robert R Alfano. Human brain cancer studied by resonance raman spectroscopy. Journal of biomedical optics, 17(11):116021, 2012.

[3] Nicola Coluccelli, Vikas Kumar, Marco Cassinerio, Gianluca Galzerano, Marco Marangoni, and Giulio Cerullo. Er/tm: fiber laser system for coherent raman microscopy. Optics letters, 39(11):3090-3093, 2014.

[4] Francesco Crisafi, Vikas Kumar, Tullio Scopigno, Marco Marangoni, Giulio Cerullo, and Dario Polli. In-line balanced detection stimulated raman scattering microscopy. Scientific reports, 7(1):10745, 2017.

[5] M. Carminati, G. Gervasoni, M. Sampietro, and G. Ferrari. Note: Differential configurations for the mitigation of slow fluctuations limiting the resolution of digital lock-in amplifiers. Review of Scientific Instruments, 87(2):026102, feb 2016.

[6] Alejandro De la Cadena, Carlo M Valensise, Marco Marangoni, Giulio Cerullo, and Dario Polli. Broadband stimulated raman scattering microscopy with wavelength-scanning detection. Journal of Raman Spectroscopy.

[7] Keisuke Seto, Yoshinao Okuda, Eiji Tokunaga, and Takayoshi Kobayashi. Development of a multiplex stimulated raman microscope for spectral imaging through multi-channel lock-in detection. Review of Scientific Instruments, 84(8):083705, 2013.

[8] Camille Scotté, Siddharth Sivankutty, Patrick Stockton, Randy A Bartels, and Hervé Rigneault. Compressive raman imaging with spatial frequency modulated illumination. Optics letters, 44(8):1936-1939, 2019.

[9] Benjamin Figueroa, Walter Fu, Tai Nguyen, Kseniya Shin, Bryce Manifold, Frank Wise, and Dan Fu. Broadband hyperspectral stimulated raman scattering microscopy with a parabolic fiber amplifier source. Biomedical optics express, 9(12):6116-6131, 2018.

[10] Chien-Sheng Liao, Mikhail N Slipchenko, Ping Wang, Junjie Li, SeungYoung Lee, Robert A Oglesbee, and Ji-Xin Cheng. Microsecond scale vibrational spectroscopic imaging by multiplex stimulated raman scattering microscopy. Light: Science \& Applications, 4(3):e265, 2015. 